\title{
Explicit Ramsey graphs and Erdős distance problems over finite Euclidean and non-Euclidean spaces
}

\author{
Le Anh Vinh \\ Mathematics Department \\ Harvard University \\ Cambridge, MA 02138, US \\ vinh@math.harvard.edu
}

Submitted: Nov 21, 2007; Accepted: Dec 17, 2007; Published: Jan 1, 2008

Mathematics Subject Classifications: 05C35, 05C38, 05C55, 05C25

\begin{abstract}
We study the Erdös distance problem over finite Euclidean and non-Euclidean spaces. Our main tools are graphs associated to finite Euclidean and non-Euclidean spaces that are considered in Bannai-Shimabukuro-Tanaka $(2004,2007)$. These graphs are shown to be asymptotically Ramanujan graphs. The advantage of using these graphs is twofold. First, we can derive new lower bounds on the Erdös distance problems with explicit constants. Second, we can construct many explicit tough Ramsey graphs $R(3, k)$.
\end{abstract}

\section{Introduction}

Let $\mathbb{F}_{q}$ denote the finite field with $q$ elements where $q \gg 1$ is an odd prime power. Let $E \subset \mathbb{F}_{q}^{d}, d \geqslant 2$. Then the analogue of the classical Erdös distance problem is to determine the smallest possible cardinality of the set

$$
\Delta(E)=\left\{|x-y|^{2}=\left(x_{1}-y_{1}\right)^{2}+\ldots+\left(x_{d}-y_{d}\right)^{2}: x, y \in E\right\},
$$

viewed as a subset of $\mathbb{F}_{q}$. Suppose that -1 is a square in $\mathbb{F}_{q}$, then using spheres of radius 0 , there exists a set of cardinality precisely $q^{d / 2}$ such that $\Delta(E)=\{0\}$. Thus, we only consider the set $E \subset \mathbb{F}_{q}^{d}$ of cardinality $C q^{\frac{q}{2}+\varepsilon}$ for some constant $C$. Bourgain, Katz and Tao ([11]) showed, using intricate incidence geometry, that for every $\varepsilon>0$, there exists $\delta>0$, such that if $E \in \mathbb{F}_{q}^{2}$ and $|E| \leqslant C_{\epsilon} q^{2-\epsilon}$, then $|\Delta(E)| \geqslant C_{\delta} q^{\frac{1}{2}+\delta}$ for some constants $C_{\epsilon}, C_{\delta}$. The relationship between $\varepsilon$ and $\delta$ in their argument is difficult to determine. Going up to higher dimension using arguments of Bourgain, Katz and Tao is quite subtle. Iosevich and Rudnev ([18]) establish the following results using Fourier analytic methods. 
Theorem 1 ([18]) Let $E \subset \mathbb{F}_{q}^{d}$ such that $|E| \gtrsim C q^{d / 2}$ for $C$ sufficient large. Then

$$
|\Delta(E)| \gtrsim \min \left\{q, \frac{|E|}{q^{\frac{d-1}{2}}}\right\} .
$$

By modifying the proof of Theorem 1 slightly, Iosevich and Rudnev ([18]) obtain the following stronger conclusion.

Theorem 2 ([18]) Let $E \subset \mathbb{F}_{q}^{d}$ such that $|E| \geqslant C q^{\frac{d+1}{2}}$ for sufficient large constant $C$. Then for every $t \in \mathbb{F}_{q}$ there exist $x, y \in E$ such that $|x-y|^{2}=t$. In other words, $|\Delta(E)|=q$.

It is, however, more natural to define the analogues of Euclidean graphs for each nondegenerate quadratic from on $V=\mathbb{F}_{q}^{d}, d \geqslant 2$. Let $Q$ be a non-degenerate quadratic form on $V$. For any $E \subset V$, we define the distance set of $E$ with respect to $Q$ :

$$
\Delta_{Q}(E)=\{Q(x-y): x, y \in E\}
$$

viewed as a subset of $\mathbb{F}_{q}$. Our first result is the following.

Theorem 3 Let $Q$ be a non-degenerate quadratic from on $\mathbb{F}_{q}^{d}$, $d \geqslant 2$. Let $E \subset \mathbb{F}_{q}^{d}$ such that $|E| \geqslant 3 q^{\frac{d}{2}+\varepsilon}$ for some $\varepsilon>0$, then

$$
\left|\Delta_{Q}(E)\right| \geqslant \min \left\{\frac{|E|}{3 q^{(d-1) / 2}}, q\right\}
$$

for $q \gg 1$.

This result is not new. It follows from the same proof as the proofs of Theorem 1 and Theorem 2 in [18]. It is also explicitly proved in [17]. We provide here a different proof for this result.

An interesting question is to study the analogue of the Erdős distance problem in nonEuclidean spaces. In order to make this paper concise, we will only consider the Erdös distance problem in the finite non-Euclidean plane (or so-called the finite upper half plane). In Section 2, we will see how to obtain various finite non-Euclidean spaces from the action of classical Lie groups on the set of non-isotropic points, lines and hyperplanes. Most of our results in this paper hold in this more general setting. We will address these results in a subsequent paper.

The well-known finite upper half plane is constructed in a similar way using an analogue of Poincaré's non-Euclidean distance. We follow the construction in [28]. Let $\mathbb{F}_{q}$ be the finite field with $q=p^{r}$ elements, where $p$ is an odd prime. Suppose $\sigma$ is a generator of the multiplicative group $\mathbb{F}_{q}^{*}$ of nonzero elements in $\mathbb{F}_{\mathbb{q}}$. The extension $\mathbb{F}_{q} \cong \mathbb{F}_{q}(\sigma)$ is analogous to $\mathbb{C}=\mathbb{R}[i]$. We define the finite Poincaré upper half-plane as

$$
H_{q}=\left\{z=x+y \sqrt{\sigma}: x, y \in \mathbb{F}_{q} \text { and } y \neq 0\right\} .
$$


Note that "half-plane" is something of a misnomer since $y \neq 0$ may not be a good finite analogue of the condition $y>0$ that defines the usual Poincaré upper half-plane in $C$. In fact, $H_{q}$ is more like a double covering of a finite upper half-plane. We use the familiar notation from complex analysis for $z=x+y \sqrt{\sigma} \in H_{q}: x=\operatorname{Re}(z), y=\operatorname{Im}(z)$, $\bar{z}=x-y \sqrt{\sigma}=z^{q}, N(z)=$ Norm of $z=z \bar{z}=z^{1+q}$. The Poincaré distance between $z, w \in H_{q}$ is

$$
d(z, w)=\frac{N(z-w)}{\operatorname{Im}(z) \operatorname{Im}(w)} \in \mathbb{F}_{q} .
$$

This distance is not a metric in the sense of analysis, but it is $G L\left(2, \mathbb{F}_{q}\right)$-invariant: $d(g z, g w)=d(z, w)$ for all $g \in G L\left(2, \mathbb{F}_{q}\right)$ and all $z, w \in H_{q}$. Let $E \subset H_{q}$. We define the distance set with respect to the Poincaré distance:

$$
\Delta_{H}(E)=\{d(x, y): x, y \in E\}
$$

viewed as a subset of $\mathbb{F}_{q}$. The following result is a non-Euclidean analogue of Theorem 3 .

Theorem 4 Let $E \subset H_{q}$ such that $|E| \geqslant 3 q^{\frac{1}{2}+\varepsilon}$ for some $\varepsilon>0$, then

$$
\left|\Delta_{H}(E)\right| \geqslant \min \left\{\frac{|E|}{3 q^{1 / 2}}, q-1\right\}
$$

for $q \gg 1$.

We also have the Erdös problem for two sets. Let $E, F \subset \mathbb{F}_{q}^{d}, d \geqslant 2$. Given a nondegenerate quadratic $Q$ form on $\mathbb{F}_{q}^{d}$. We define the set of distances between two sets $E$ and $F$ :

$$
\Delta_{Q}(E, F)=\{Q(x, y): x \in E, y \in F\} .
$$

We will prove the following analogues of Theorem 3 for the distance set $\Delta_{Q}(E, F)$.

Theorem 5 Let $E, F \subset \mathbb{F}_{q}^{d}$ such that $|E||F| \geqslant 9 q^{(d-1)+\epsilon}$ for some $\varepsilon>0$, then

$$
\Delta_{Q}(E, F) \geqslant \min \left\{\frac{\sqrt{|E||F|}}{3 q^{(d-1) / 2}}, q\right\}
$$

for $q \gg 1$.

In finite upper half plane, we define the set of distances between two sets $E, F \subset H_{q}$ :

$$
\Delta_{H}(E, F)=\{d(x, y): x \in E, y \in F\}
$$

where $d(x, y)$ is the finite Poincaré distance between $x$ and $y$. Similarly, we have an analogue of Theorem 4 for the distance set $\Delta_{H}(E, F)$. 
Theorem 6 Let $E, F \subset H_{q}$ such that $|E||F| \geqslant 9 q^{1+2 \epsilon}$ for some $\varepsilon>0$, then

$$
\Delta_{H}(E, F) \geqslant \min \left\{\frac{\sqrt{|E||F|}}{3 q^{1 / 2}}, q-1\right\}
$$

for $q \gg 1$.

Note that Theorem 5 is also not new. It follows instantly from incidence bounds in Theorem 3.4 in [17] as going from a one set formulation in the Fourier proofs in [17] to a two set formulation is just a matter of inserting a different letter in couple of places. The proof we present in this paper however is new.

The rest of this paper is organized as follows. In Section 2 we construct our main tools to study the Erdős problem over finite Euclidean and non-Euclidean spaces, the finite Euclidean and non-Euclidean graphs. Our construction follows one of Bannai, Shimabukuro and Tanaka in $[8,7]$. In Section 3 we establish some useful facts about these finite graphs. One important result is for infinitely many values of $q$, these graphs disprove a conjecture of Chvatál and also provide a good lower bound for the Ramsey number $R(3, k)$. We then prove our main results, Theorems 3, 4, 5 and 6, in Section 4. In the last section, we will discuss the similarities of our approach and those in [17] and [18].

We also call the reader's attention to the fact that the application of the spectral method from graph theory in sum-product estimates and Erdős distance problem was independently used by $\mathrm{Vu}$ in [32].

\section{Finite Euclidean and non-Euclidean Graphs}

In this section, we summarise main results from Bannai-Shimabukuro-Tanaka $[7,8]$. We follow their constructions of finite Euclidean and non-Euclidean graphs.

Let $Q$ be a non-degenerate quadratic form on $V$. We define the corresponding bilinear from on $V$ :

$$
\langle x, y\rangle_{Q}=Q(x+y)-Q(x)-Q(y) .
$$

Let $O(V, Q)$ be the group of all linear transformations on $V$ that fix $Q$ (which is called the orthogonal group associated with the quadratic form $Q$ ). The non-degenerate quadratic forms over $\mathbb{F}_{q}^{n}$ are classified as follows:

1. Suppose that $n=2 m$. If $q$ odd then there are two inequivalent non-degenerate quadratic forms $Q_{2 m}^{+}$and $Q_{2 m}^{-}$:

$$
\begin{aligned}
& Q_{2 m}^{+}(x)=2 x_{1} x_{2}+\ldots+2 x_{2 m-1} x_{2 m}, \\
& Q_{2 m}^{-}(x)=2 x_{1} x_{2}+\ldots+2 x_{2 m-3} x_{2 m-2}+x_{2 m-1}^{2}-\alpha x_{2 m}^{2},
\end{aligned}
$$

where $\alpha$ is a non-square element in $\mathbb{F}_{q}$. If $q$ even then there are also two inequivalent non-degenerate quadratic forms $Q^{+}$and $Q^{-}$:

$$
\begin{aligned}
& Q_{2 m}^{+}(x)=x_{1} x_{2}+\ldots+x_{2 m-1} x_{2 m} \\
& Q_{2 m}^{-}(x)=x_{1} x_{2}+\ldots+x_{2 m-3} x_{2 m-2}+x_{2 m-1}^{2}+\beta x_{2 m}^{2}
\end{aligned}
$$


where $\beta$ is an element in $\mathbb{F}_{q}$ such that the polynomial $t^{2}+t+\beta$ is irreducible over $\mathbb{F}_{q}$. We write $O_{2 m}^{+}=O\left(V, Q_{2 m}^{+}\right)$and $O_{2 m}^{-}=O\left(V, Q_{2 m}^{-}\right)$.

2. Suppose that $n=2 m+1$ is odd. If $q$ is odd, then there are two inequivalent nondegenerate quadratic forms $Q_{2 m+1}$ and $Q_{2 m+1}^{\prime}$ :

$$
\begin{aligned}
& Q_{2 m+1}(x)=2 x_{1} x_{2}+\ldots+2 x_{2 m-1} x_{2 m}+x_{2 m+1}^{2}, \\
& Q_{2 m+1}^{\prime}(x)=2 x_{1} x_{2}+\ldots+2 x_{2 m-1} x_{2 m}+\alpha x_{2 m-1}^{2},
\end{aligned}
$$

where $\alpha$ is a non-square element in $\mathbb{F}_{q}$. But the groups $O\left(V, Q_{2 m+1}\right)$ and $O\left(V, Q_{2 m+1}^{\prime}\right)$ are isomorphic. If $q$ is even then there exists exactly one inequivalent non-degenerate quadratic form $Q_{2 m+1}$ :

$$
Q_{2 m+1}(x)=x_{1} x_{2}+\ldots+x_{2 m-1} x_{2 m}+x_{2 m+1}^{2} .
$$

In this case, we write $O_{2 m+1}=O\left(V, Q_{2 m+1}\right)$.

\subsection{Finite Euclidean Graphs}

Let $Q$ be a non-degenerate quadratic form on $V$. Then the finite Euclidean graph $E_{q}(n, Q, a)$ is defined as the graph with vertex set $V$ and the edge set

$$
E=\{(x, y) \in V \times V \mid x \neq y, Q(x-y)=a\} .
$$

In [8], Bannai, Shimabukuro and Tanaka showed that the finite Euclidean graphs $E_{q}(n, Q, a)$ are not always Ramanujan. Fortunately, they are always asymptotically Ramanujan. The following theorem summaries (in a rough form) the results from Sections 2-6 in [8] and Section 3 in Kwok [22].

Theorem 7 Let $\rho$ be a primitive element of $\mathbb{F}_{q}$.

a) The graphs $E_{q}\left(2 m, Q_{2 m}^{ \pm}, \rho^{i}\right)$ are regular of valency $k=q^{2 m-1} \pm q^{m-1}$ for $1 \leqslant i \leqslant q-1$. Let $\lambda$ be any eigenvalue of the graph $E_{q}\left(2 m, Q_{2 m}^{ \pm}, \rho^{i}\right)$ with $\lambda \neq$ valency of the graph then

$$
|\lambda| \leqslant 2 q^{(2 m-1) / 2}
$$

b) The graphs $E_{q}\left(2 m+1, Q_{2 m+1}, \rho^{i}\right)$ are regular of valency $k=q^{2 m} \pm q^{m}$ for $1 \leqslant i \leqslant q-1$. Let $\lambda$ be any eigenvalue of the graph $E_{q}\left(2 m+1, Q_{2 m+1}, \rho^{i}\right)$ with $\lambda \neq$ valency of the graph then

$$
|\lambda| \leqslant 2 q^{m}
$$

\subsection{Finite non-Euclidean Graphs}

In order to keep this paper concise, we will restrict our discussion to the finite nonEuclidean graphs obtained from the action of the simple orthogonal group on the set of non-isotropic points. Similar results hold for graphs obtained from the action of various Lie groups on the set of non-isotropic points, lines and hyperplanes. We will address these results in a subsequent paper. 


\subsubsection{Graphs obtained from the action of simple orthogonal group $O_{2 m+1}(q)$ ( $q$ odd) on the set of non-isotropic points}

Let $V=\mathbb{F}_{q}^{2 m+1}$ be the $(2 m+1)$-dimensional vector space over the finite field $\mathbb{F}_{q}$ ( $q$ is an odd prime power). For each element $x$ of $V$, we denote the 1-dimensional subspace containing $x$ by $[x]$. Let $\Theta, \Omega$ be the set of all square type and the set of all non-square-type non-isotropic 1-dimensional subspaces of $V$ with respect to the quadratic form $Q_{2 m+1}$, respectively. Then we have $|\Theta|=\left(q^{2 m}-q^{m}\right) / 2$ and $|\Omega|=\left(q^{2 m}+q^{m}\right) / 2$. The simple orthogonal group $O_{2 m+1}(q)$ acts transitively on $\Theta$ and $\Omega$.

We define the graphs $H_{q}\left(O_{2 m+1}, \Theta, i\right)$ (for $1 \leqslant i \leqslant(q+1) / 2$ ) as follows (let $E_{i}$ be the edge set of $\left.H_{q}\left(O_{2 m+1}, \Theta, i\right)\right)$ :

$$
\begin{aligned}
([x],[y]) \in E_{1} & \Leftrightarrow\left(\begin{array}{l}
x \\
y
\end{array}\right) . S .\left(\begin{array}{l}
x \\
y
\end{array}\right)^{t}=\left(\begin{array}{cc}
\nu & 1 \\
1 & \nu^{-1}
\end{array}\right), \\
([x],[y]) \in E_{i} & \Leftrightarrow\left(\begin{array}{l}
x \\
y
\end{array}\right) . S .\left(\begin{array}{l}
x \\
y
\end{array}\right)^{t}=\left(\begin{array}{cc}
\nu & 1 \\
1 & \nu^{2 i-3}
\end{array}\right),(2 \leqslant i \leqslant(q-1) / 2) \\
([x],[y]) \in E_{(q+1) / 2} & \Leftrightarrow\left(\begin{array}{l}
x \\
y
\end{array}\right) . S .\left(\begin{array}{l}
x \\
y
\end{array}\right)^{t}=\left(\begin{array}{cc}
\nu & 0 \\
0 & \nu
\end{array}\right),
\end{aligned}
$$

where $\nu \in \mathbb{F}_{q}$ is a primitive element of $\mathbb{F}_{q}, A^{t}$ denotes the transpose of $A$ and $S$ is the matrix of the associated bilinear form of $Q_{2 m+1}$. Note that for $m=1$ then we have the finite analogue $H_{q}$ of the upper half plane.

We define the graph $H_{q}\left(O_{2 m+1}, \Omega, i\right)$ (for $1 \leqslant i \leqslant(q+1) / 2$ ) as follows (let $E_{i}$ be the edge set of $\left.H_{q}\left(O_{2 m+1}, \Omega, i\right)\right)$ :

$$
\begin{array}{lll}
([x],[y]) \in E_{1} & \Leftrightarrow & Q_{2 m+1}(x+y)=0, \\
([x],[y]) \in E_{i} & \Leftrightarrow & Q_{2 m+1}(x+y)=2+2 \nu^{-(i-1)},(2 \leqslant i \leqslant(q-1) / 2) \\
([x],[y]) \in E_{(q+1) / 2} & \Leftrightarrow & Q_{2 m+1}(x+y)=2,
\end{array}
$$

where we assume $Q_{2 m+1}(x)=1$ for all $[x] \in \Omega$.

As in finite Euclidean case, the graphs obtained in this section are always asymptotically Ramanujan. The following theorem summaries the results from Sections 1 and 2 in [7] and from Section 7 in [5].

Theorem 8 a) The graphs $H_{q}\left(O_{2 m+1}, \Theta, i\right)(1 \leqslant i \leqslant(q-1) / 2)$ are regular of valency $q^{2 m-1} \pm q^{m-1}$. The graph $H_{q}\left(O_{2 m+1}, \Theta,(q+1) / 2\right)$ is regular of valency $\left(q^{2 m-1} \pm q^{m-1}\right) / 2$. Let $\lambda$ be any eigenvalue of the graph $H_{q}\left(O_{2 m+1}, \Theta, i\right)$ with $\lambda \neq$ valency of the graph then

$$
|\lambda| \leqslant 2 q^{(2 m-1) / 2} \text {. }
$$

b) The graphs $H_{q}\left(O_{2 m+1}, \Omega, i\right)(1 \leqslant i \leqslant(q-1) / 2)$ are regular of valency $\left.q^{2 m-1} \pm q^{m-1}\right)$. The graph $H_{q}\left(O_{2 m+1}, \Omega,(q+1) / 2\right)$ is regular of valency $\left(q^{2 m-1} \pm q^{m-1}\right) / 2$. Let $\lambda$ be any eigenvalue of the graph $H_{q}\left(O_{2 m+1}, \Omega, i\right)$ with $\lambda \neq$ valency of the graph then

$$
|\lambda| \leqslant 2 q^{(2 m-1) / 2} .
$$




\subsubsection{Graphs obtained from the action of simple orthogonal group $O_{2 m}^{ \pm}(q)(q$ odd) on the set of non-isotropic points}

Let $V=\mathbb{F}_{q}^{2 m}$ be the $2 m$-dimensional vector space over the finite field $\mathbb{F}_{q}$ ( $q$ is an odd prime power). For each element $x$ of $V$, we denote the 1-dimensional subspace containing $x$ by $[x]$. Let $\Omega_{1}, \Omega_{2}$ be the set of all square type and the set of all non-square-type non-isotropic 1-dimensional subspaces of $V$ with respect to the quadratic form $Q_{2 m}^{+}$, respectively. Then we have $\left|\Omega_{1}\right|=\left|\Omega_{2}\right|=\left(q^{2 m-1}-q^{m-1}\right) / 2$. The orthogonal group $O_{2 m}^{+}(q)$ with respect to the quadratic from $Q_{2 m}^{+}$over $\mathbb{F}_{q}$ acts on both $\Omega_{1}$ and $\Omega_{2}$ transitively. We define the graph $H_{q}\left(O_{2 m}^{+}, \Omega_{1}, i\right)$ (for $\left.1 \leqslant i \leqslant(q+1) / 2\right)$ as follows (let $E_{i}$ be the edge set of $H_{q}\left(O_{2 m}^{+}, \Omega_{1}, i\right)$ ):

$$
\begin{array}{ll}
([x],[y]) \in E_{i} & \Leftrightarrow\langle x, y\rangle_{Q_{2 m}^{+}}=2^{-1} \nu^{i},(1 \leqslant i \leqslant(q-1) / 2) \\
([x],[y]) \in E_{(q+1) / 2} & \Leftrightarrow\langle x, y\rangle_{Q_{2 m}^{+}}=0,
\end{array}
$$

where we assume $Q_{2 m}^{+}(x)=1$ for all $[x] \in \Omega$.

Let $\Theta_{1}, \Theta_{2}$ be the set of all square type and the set of all non-square-type non-isotropic 1-dimensional subspaces of $V$ with respect to the quadratic form $Q_{2 m}^{-}$, respectively. Then we have $\left|\Theta_{1}\right|=\left|\Theta_{2}\right|=\left(q^{2 m-1}+q^{m-1}\right) / 2$. The orthogonal group $O_{2 m}^{-}(q)$ with respect to the quadratic from $Q_{2 m}^{-}$over $\mathbb{F}_{q}$ acts on both $\Theta_{1}$ and $\Theta_{2}$ transitively. We define the graph $H_{q}\left(O_{2 m}^{-}, \Theta_{1}, i\right)($ for $1 \leqslant i \leqslant(q+1) / 2)$ as follows (let $E_{i}$ be the edge set of $H_{q}\left(O_{2 m}^{-}, \Omega_{1}, i\right)$ ):

$$
\begin{array}{ll}
([x],[y]) \in E_{i} & \Leftrightarrow\langle x, y\rangle_{Q_{2 m}^{-}}=2^{-1} \nu^{i},(1 \leqslant i \leqslant(q-1) / 2) \\
([x],[y]) \in E_{(q+1) / 2} & \Leftrightarrow \quad\langle x, y\rangle_{Q_{2 m}^{-}}=0,
\end{array}
$$

where we assume $Q_{2 m}^{-}(x)=1$ for all $[x] \in \Omega$.

The graphs obtained in this section are always asymptotically Ramanujan. The following theorem summaries the results from Sections 4 and 5 in [7] and from Section 4 in [5].

Theorem 9 a) The graphs $H_{q}\left(O_{2 m}, \Theta_{1}, i\right)(1 \leqslant i \leqslant(q-1) / 2)$ are regular of valency $q^{2 m-2} \pm q^{m-1}$. The graph $H_{q}\left(O_{2 m}, \Theta,(q+1) / 2\right)$ is regular of valency $\left(q^{2 m-2} \pm q^{m-1}\right) / 2$. Let $\lambda$ be any eigenvalue of the graph $H_{q}\left(O_{2 m}, \Theta, i\right)$ with $\lambda \neq$ valency of the graph then

$$
|\lambda| \leqslant 2 q^{(2 m-2) / 2} .
$$

b) The graphs $H_{q}\left(O_{2 m}, \Omega_{1}, i\right)(1 \leqslant i \leqslant(q-1) / 2)$ are regular of valency $q^{2 m-2} \pm q^{m-1}$. The graph $H_{q}\left(O_{2 m+1}, \Omega,(q+1) / 2\right)$ is regular of valency $\left(q^{2 m-2} \pm q^{m-1}\right) / 2$. Let $\lambda$ be any eigenvalue of the graph $H_{q}\left(O_{2 m}, \Omega_{1}, i\right)$ with $\lambda \neq$ valency of the graph then

$$
|\lambda| \leqslant 2 q^{(2 m-2) / 2} .
$$

\section{$3 \quad$ Explicit Tough Ramsey Graphs}

We call a graph $G=(V, E)(n, d, \lambda)$-regular if $G$ is a $d$-regular graph on $n$ vertices with the absolute value of each of its eigenvalues but the largest one is at most $\lambda$. It is well-known that if $\lambda \ll d$ then a $(n, d, \lambda)$-regular graph behaves similarly as a random graph $G_{n, d / n}$. Presicely, we have the following result (see Corollary 9.2.5 and Corollary 9.2.6 in [3]). 
Theorem 10 ([3]) Let $G$ be a $(n, d, \lambda)$-regular graph.

a) For every set of vertices $B$ and $C$ of $G$, we have

$$
\left|e(B, C)-\frac{d}{n}\right| B|| C|| \leqslant \lambda \sqrt{|B||C|}
$$

where $e(B, C)$ is the number of edges in the induced subgraph of $G$ on $B$ (i.e. the number of ordered pairs $(u, v)$ where $u \in B, v \in C$ and $u v$ is an edge of $G$ ).

b) For every set of vertices $B$ of $G$, we have

$$
\left.\left|e(B)-\frac{d}{2 n}\right| B\right|^{2}\left|\leqslant \frac{1}{2} \lambda\right| B \mid,
$$

where $e(B)$ is number of edges in the induced subgraph of $G$ on $B$.

Let $B, C$ be one of the maximum independent pairs of $G$, i.e. the "bipartite" subgraph induced on $(B, C)$ are empty and $|B||C|$ is maximum. Let $\alpha_{2}(G)$ denote the size $|B||C|$ of this pair. Then from (7), we have

$$
\alpha_{2}(G) \leqslant \frac{\lambda^{2} n^{2}}{d^{2}}
$$

Let $B$ be one of the maximum independent sets of $G$. Then from (8), we have

$$
\alpha(G)=|B| \leqslant \frac{n \lambda}{d}
$$

and

$$
\chi(G) \geqslant \frac{|V(G)|}{\alpha(G)} \geqslant \frac{d}{\lambda} .
$$

The toughness $t(G)$ of a graph $G$ is the largest real $t$ so that for every positive integer $x \geq 2$ one should delete at least $t x$ vertices from $G$ in order to get an induced subgraph of it with at least $x$ connected components. $G$ is $t$-tough if $t(G) \geq t$. This parameter was introduced by Chvatál in [12]. Chvatál also conjectures the following: there exists an absolute constant $t_{0}$ such that every $t_{0}$-tough graph is pancyclic. This conjecture was disproved by Bauer, van den Heuvel and Schmeichel [9] who constructed, for every real $t_{0}$, a $t_{0}$-tough triangle-free graph. They define a sequence of triangle-free graphs $H_{1}, H_{2}, H_{3}, \ldots$ with $\left|V\left(H_{j}\right)\right|=2^{2 j-1}(j+1)$ ! and $t\left(H_{j}\right) \geq \sqrt{2 j+4} / 2$. To bound the toughness of a $(n, d, \lambda)$-regular graph, we have the following result which is due to Alon in [2].

Theorem 11 [2] Let $G=(V, E)$ be an $(n, d, \lambda)$-graph. Then the toughness $t=t(G)$ of $G$ satisfies

$$
t>\frac{1}{3}\left(\frac{d^{2}}{\lambda d+\lambda^{2}}-1\right)
$$


Let $G$ be any graph of the form $E_{q}\left(2 m, Q_{2 m}^{ \pm}, a\right), E_{q}\left(2 m+1, Q_{2 m+1}, a\right), H_{q}(2 m+$ $1, \Theta, i), H_{q}(2 m+1, \Omega, i), H_{q}\left(2 m, \Omega_{1}, i\right)$ and $H_{q}\left(2 m, \Theta_{1}, i\right)$ for $a \neq 0 \in \mathbb{F}_{q}$ and $1 \leqslant i \leqslant$ $(q+1) / 2$. Then from Theorems 7,8 and 9 , the graph $G$ is $\left(c_{1} q^{n}+O\left(q^{n / 2}\right), c_{2} q^{n-1}+\right.$ $\left.O\left(q^{(n-1) / 2}\right), 2 q^{(n-1) / 2}\right)$-regular for some $n \geqslant 2$ and $c_{1}, c_{2} \in\left\{\frac{1}{2}, 1\right\}$. By (10), (11) and (12), we can show that the finite Euclidean and non-Euclidean graphs have high chromatic number, small independent number and high tough number.

Theorem 12 Let $G$ be any graph of the form $E_{q}\left(2 m, Q_{2 m}^{ \pm}, a\right), E_{q}\left(2 m+1, Q_{2 m+1}, a\right)$, $H_{q}(2 m+1, \Theta, i), H_{q}(2 m+1, \Omega, i), H_{q}\left(2 m, \Omega_{1}, i\right)$ and $H_{q}\left(2 m, \Theta_{1}, i\right)$ for $a \neq 0 \in \mathbb{F}_{q}$ and $1 \leqslant i \leqslant(q+1) / 2$. Suppose that $|V(G)|=c q^{n}+O\left(q^{(n-1) / 2}\right)$.

1. The independent number of $G$ is small: $\alpha(G) \leqslant(4+o(1))|V(G)|^{(n+1) / 2 n}$.

2. The chromatic number of $G$ is high: $\chi(G) \geqslant|V(G)|^{(n-1) / 2 n} /(4+o(1))$.

3. The toughness of $G$ is at least $|V(G)|^{(n-1) / 2 n} /(12+o(1))$.

In [31], the authors derived the following theorem using only elementary algebra. This theorem can also be derived from character tables of the association schemes of affine type ([22]) and of finite orthogonal groups acting on the nonisotropic points ([5]).

Theorem 13 Among all finite Euclidean and non-Euclidean graphs, the only triangle-free graphs are

1. $E_{q}\left(2, Q^{-}, a\right)$ where 3 is square in $\mathbb{F}_{q}$.

2. $E_{q}\left(2, Q^{+}, a\right)$ where 3 is nonsquare in $\mathbb{F}_{q}$.

3. $H_{q}(3, Q, a)$ for at least one element $a \in \mathbb{F}_{q}^{*}$.

Theorems 12 and 13 shows that the finite Euclidean $E_{q}\left(2, Q^{+}, a\right)$, where $q$ is a prime of form $q=12 k \pm 5$ and $a \neq 0 \in \mathbb{F}_{q}$, is an explicit triangle-free graph on $n_{q}=q^{2}$ vertices whose chromatic number exceeds $0.5 n_{q}^{1 / 4}$. Therefore, this disproves the conjecture of Chavatál. In addition, this graph is an explicit construction showing that $R(3, k) \geq$ $\Omega\left(k^{4 / 3}\right)$.

Note that, in [24], the authors constructed explicitly for every $d=p+1$ where $p \equiv 1$ ( $\bmod 4)$ is a prime, and for every $n=q\left(q^{2}-1\right) / 2$ where $q \equiv 1(\bmod 4)$ is a prime and $p$ is a quadratic residue modulo $q,(n, d, \lambda)$ graphs $G_{n}$ with $\lambda=2 \sqrt{d-1}$, where the grith of $G_{n}$ is at least $2 \log _{p} q \geqslant \frac{2}{3} \log _{d-1} n$. Using Theorem 11, Noga Alon [2] derived the existence of $t_{0}$-tough graphs without cycles of length up to $c\left(t_{0}\right) \log n$, for an arbitrary constant $t_{0}$.

Moreover, the bounds obtained from Theorems 12 and 13 match with the bounds obtained by code graphs in Theorem 3.1 in [2]. These graphs are Caley graphs and their construction is based on some of the properties of certain Dual BCH error-correcting codes. For a positive integer $k$, let $F_{k}=G F\left(2^{k}\right)$ denote the finite field with $2^{k}$ elements. The elements of $F_{k}$ are represented by binary vectors of length $k$. If $a$ and $b$ are two such vectors, let $(a, b)$ denote their concatenation. Let $G_{k}$ be the graph whose vertices are all 
$n=2^{2 k}$ binary vectors of length $2 k$, where two vectors $u$ and $v$ are adjacent if and only if there exists a non-zero $z \in F_{k}$ such that $u+v=\left(z, z^{3}\right) \bmod 2$ where $z^{3}$ is computed in the field $F_{k}$. Then $G_{k}$ is a $d_{k}=2^{k}-1$-regular graph on $n_{k}=2^{2 k}$. Moreover, $G_{k}$ is triangle-free with independence number at most $2 n^{3 / 4}$. Noga Alon gives a better bound $R(m, 3) \geq \Omega\left(m^{3 / 2}\right)$ i n [1] by considering a graph with vertex set of all $n=2^{3 k}$ binary vectors of length $3 k$ (instead of all binary vectors of length $2 k$ ). Suppose that $k$ is not divisible by 3 . Let $W_{0}$ be the set of all nonzero elements $\alpha \in F_{k}$ such that the leftmost bit in the binary representation of $\alpha^{7}$ is 0 , and let $W_{1}$ be the set of all nonzero elements $\alpha \in F_{k}$ for which the leftmost bit of $\alpha^{7}$ is 1 . Then $\left|W_{0}\right|=2^{k-1}-1$ and $\left|W_{1}\right|=2^{k-1}$. Let $G_{n}$ be the graph whose vertices are all $n=2^{3 k}$ binary vectors of length $3 k$, where two vectors $u$ and $v$ are adjacent if and only if there exist $w_{0} \in W_{0}$ and $w_{1} \in W_{1}$ such that $u+v=\left(w_{0}, w_{0}^{3}, w_{0}^{5}\right)+\left(w_{1}, w_{1}^{3}, w_{1}^{5}\right)$ where the powers are computed in the field $F_{k}$ and the addition is addition module 2 . Then $G_{n}$ is a $d_{n}=2^{k-1}\left(2^{k-1}-1\right)$-regular graph on $n=2^{3 k}$ vertices. Moreover, $G_{n}$ is a triangle-free graph with independence number at most $(36+o(1)) n^{2 / 3}$. The problem of finding better bounds for the chromatic number of finite Euclidean and non-Euclidean graphs on the plane and the upper half plane, respectively touches on an important question in graph theory: what is the greatest possible chromatic number for a triangle-free regular graph of order $n$ ? It is known that if $G$ is a trianglefree graph of order $n$ then $\chi(G) \leqslant c \sqrt{n / \log n}$ (see Lemma 2 in [13]). When we drop the regularity assumption then the upper bound is best possible as Kim [21] proved the existence of a graph $G$ with order $n$ and $\chi(G) \geqslant c \sqrt{n / \log n}$. The final remark at the end of Section 5 gives us a plausible reason to conjecture that the anwer for the regular case is also $\Theta(\sqrt{n / \log n})$.

\section{Erdős distance problem}

\subsection{Proof of Theorem 3}

Let $Q$ be any non-degenerate quadratic of $\mathbb{F}_{q}^{n}$. Recall that the Euclidean graph $E_{q}(d, Q, a)$ was defined as the graph with vertex set $V$ and edge set

$$
E=\{(x, y) \in V \times V \mid x \neq y, Q(x-y)=a\} .
$$

Lemma 1 Let $E \subset \mathbb{F}_{q}^{d}$ such that $|E| \geqslant 3 q^{\frac{d+1}{2}}$. Then $\Delta_{Q}(E)=\mathbb{F}_{q}$.

Proof By Theorem 7, each graph $E_{q}(d, Q, a)$ is a $\left(q^{d}, q^{d-1} \pm q^{\lfloor(d-1) / 2\rfloor}, 2 q^{(d-1) / 2}\right)$-regular graph. By $(10)$, for any $a \neq 0 \in \mathbb{F}_{q}$, we have

$$
\alpha\left(E_{q}(d, Q, a)\right) \leqslant \frac{2 q^{(3 d-1) / 2}}{q^{d-1}-q^{(d-1) / 2}} \leqslant 3 q^{(d+1) / 2} .
$$

Thus, if $|E| \geqslant 3 q^{\frac{d+1}{2}}$ then $E$ is not an independent set of $E_{q}(d, Q, a)$, or equivalently there exist $x, y \in E$ such that $Q(x-y)=a$ for any $a \in \mathbb{F}_{q}$. This concludes the proof of the lemma. 
Lemma 2 For any $0<\varepsilon<1 / 2$. Let $E \subset \mathbb{F}_{q}$ such that $|E| \geqslant 3 q^{\frac{d}{2}+\varepsilon}$. Then

$$
\left|\Delta_{Q}(E)\right| \geqslant q^{\frac{1}{2}+\varepsilon}
$$

for any $q \geqslant 6^{1 /(\varepsilon-1 / 2)}$.

Proof By Theorem 7, each graph $E_{q}(d, Q, a)$ is a $\left(q^{d}, q^{d-1} \pm q^{\lfloor(d-1) / 2\rfloor}, 2 q^{(d-1 /) 2}\right)$-regular graph. By (10), the number of edges of $E_{q}(d, Q, a)$ in the induced subgraph on $E$ is at most

$$
e_{E_{q}(d, Q, a)}(E) \leqslant \frac{q^{d-1}+q^{(d-1) / 2}}{2 q^{d}}|E|^{2}+q^{(d-1) / 2}|E| .
$$

Suppose that $\# \Delta_{Q}(E)<q^{1 / 2+\varepsilon}$. From $(15)$, we have

$$
\begin{aligned}
\left(\begin{array}{c}
|E| \\
2
\end{array}\right) & =\sum_{a \in \Delta_{Q}(E)} e_{E_{q}(d, Q, a)}(E) \\
& <q^{1 / 2+\varepsilon}\left\{\frac{q^{d-1}+q^{(d-1) / 2}}{2 q^{d}}|E|^{2}+q^{(d-1) / 2}|E|\right\} \\
& <|E| q^{\varepsilon-\frac{1}{2}}\left\{\left(\frac{1}{2}+\frac{1}{2} q^{-(d-1) / 2}\right)|E|+q^{(d+1) / 2}\right\},
\end{aligned}
$$

which implies that

$$
\begin{aligned}
q^{\frac{1}{2}-\varepsilon}(|E|-1) & <\left(1+q^{-(d-1) / 2}\right)|E|+2 q^{(d+1) / 2} \\
& \leqslant\left(1+q^{-1 / 2}+\frac{2}{3} q^{\frac{1}{2}-\varepsilon}\right)|E|
\end{aligned}
$$

Therefore, we have

$$
\begin{aligned}
q^{\frac{1}{2}-\varepsilon} & >\left(\frac{1}{3} q^{\frac{1}{2}-\varepsilon}-1-q^{\frac{1}{2}}\right)|E| \\
& \geqslant\left(q^{\frac{1}{2}-\varepsilon}-3-3 q^{-\frac{1}{2}}\right) q^{\frac{d}{2}+\varepsilon}
\end{aligned}
$$

which is a contradiction if $q>6^{1 /(1 / 2-\varepsilon)}$. The lemma follows.

Theorem 3 follows immediately from Lemma 1 and Lemma 2.

\subsection{Proof of Theorem 4}

For a fixed $a \in \mathbb{F}_{q}$, the finite non-Euclidean graph $V_{q}(\sigma, a)$ has vertices as the points in $H_{q}$ and edges between vertices $z, w$ if and only if $d(z, w)=a$. Except when $a=0$ or $a=4 \sigma, V_{q}(\sigma, a)$ is a connected $(q+1)$-regular graph. When $a=0,4 \sigma$ then $V_{q}(\sigma, a)$ is disconnected, with one or two nodes, respectively, per connected component. As $a$ varies, we have $q-2(q+1)$-regular graphs $V_{q}(\sigma, a)$. The question of whether these graphs are always nonisomorphic or not is still open. See [28] for a survey of spectra of Laplacians of this graph. 
Lemma 3 Let $E \subset H_{q}$ such that $|E| \geqslant 2 q^{3 / 2}$. Then $\left|\Delta_{H}(E)\right| \geqslant q-1$.

Proof Each graph $V_{q}(\sigma, a)$ (with $\left.a \neq 0,4 \sigma \in \mathbb{F}_{q}\right)$ is a $\left(q^{2}-q, q+1,2 q^{1 / 2}\right)$-regular graph. By (10), for any $a \neq 0,4 \sigma \in \mathbb{F}_{q}$, we have

$$
\alpha\left(V_{q}(\sigma, a)\right) \leqslant \frac{2\left(q^{2}-q\right) q^{1 / 2}}{q+1} \leqslant 2 q^{3 / 2} .
$$

Thus, \#E $\geqslant 2 q^{3 / 2}$ then $E$ is not an independent set of $V_{q}(\sigma, a)$ or equivalently, there exist $x, y \in E$ such that $d(x-y)=a$ for any $a \in \mathbb{F}_{q}-\{0,4 a\}$. This concludes the proof of the lemma.

Note that $V_{q}(\sigma, 4 \sigma)$ is just a disjoint union of $\left(q^{2}-q\right) / 2$ edges. So we can have a set $E \in H_{q}$ with $|E|=\left(q^{2}-q\right) / 2$ and $\Delta_{H}(E)=\mathbb{F}_{q}-\{4 \sigma\}$.

Lemma 4 For any $0<\varepsilon<1 / 2$. Let $E \subset \mathbb{F}_{q}$ such that $|E| \geqslant 3 q^{\frac{d}{2}+\varepsilon}$. Then

$$
\left|\Delta_{H}(E)\right| \geqslant q^{\frac{1}{2}+\varepsilon}
$$

for any $q \geqslant 9^{1 /(\varepsilon-1 / 2)}$.

Proof For any $a \neq 0,4 \sigma \in \mathbb{F}_{q}$, each graph $V_{q}(\sigma, a)$ is a $\left(q^{2}-q, q+1,2 q^{1 / 2}\right)$-regular graph. From Theorem 10, the number of edges of $V_{q}(\sigma, a)$ in the induced subgraph on $E$ is at most

$$
e_{V_{q}(\sigma, a)}(E) \leqslant \frac{q+1}{2\left(q^{2}-q\right)}|E|^{2}+q^{1 / 2}|E| .
$$

Suppose that $\left|\Delta_{H}(E)\right|<q^{1 / 2+\varepsilon}$. From (18), we have

$$
\begin{aligned}
\left(\begin{array}{c}
|E| \\
2
\end{array}\right) & =\sum_{a \in \Delta_{H}(E)} e_{V_{q}(\sigma, a)}(E) \\
& <q^{1 / 2+\varepsilon}\left\{\frac{q+1}{2\left(q^{2}-q\right)}|E|^{2}+q^{1 / 2}|E|\right\} \\
& <|E| q^{\varepsilon-\frac{1}{2}}\left\{\left(\frac{1}{2}+\frac{1}{q-2}\right)|E|+q^{3 / 2}\right\},
\end{aligned}
$$

which implies that

$$
\begin{aligned}
q^{\frac{1}{2}-\varepsilon}(|E|-1) & <\left(1+\frac{2}{q-2}\right)|E|+2 q^{3 / 2} \\
& \leqslant\left(1+\frac{2}{q-2}+\frac{2}{3} q^{\frac{1}{2}-\varepsilon}\right)|E|
\end{aligned}
$$

Therefore, we have

$$
\begin{aligned}
q^{\frac{1}{2}-\varepsilon} & >\left(\frac{1}{3} q^{\frac{1}{2}-\varepsilon}-1-\frac{2}{q-2}\right)|E| \\
& \geqslant\left(q^{\frac{1}{2}-\varepsilon}-3-\frac{6}{q-2}\right) q^{1+\varepsilon}
\end{aligned}
$$


which is a contradiction when $q>9^{1 /(1 / 2-\varepsilon)}$. The lemma follows.

Theorem 4 follows immediately from Lemma 3 and Lemma 4 . Similar results hold for others non-Euclidean spaces defined in Section 2. We will discuss these results in a subsequent paper.

\subsection{Set of distances between two sets}

Now we will prove Theorem 5 and Theorem 6 . For any $a \neq 0 \in \mathbb{F}_{q}$, by Theorem 10 , the number of edges of the graph $E_{q}(d, Q, a)$ in the induced "bipartite" subgraph on $(E, F)$ (two vertex parts are not necessary disjoint) is at most:

$$
e_{E_{q}(d, Q, a)} \leqslant \frac{q^{d-1}+q^{(d-1) / 2}}{q^{d}}|E||F|+2 q^{(d-1) / 2} \sqrt{|E||F|} .
$$

Thus, we have

$$
\begin{aligned}
|E||F| & =\sum_{a \in \Delta_{Q}(E, F)} e_{E_{q}(d, Q, a)} \\
& \leqslant \Delta_{Q}(E, F)\left(\frac{q^{d-1}+q^{(d-1) / 2}}{q^{d}}|E||F|+2 q^{(d-1) / 2} \sqrt{|E||F|}\right),
\end{aligned}
$$

which implies that

$$
\Delta_{Q}(E, F) \geqslant \frac{1}{\frac{1}{q}+\frac{1}{q^{(d+1) / 2}}+\frac{2 q^{(d-1) / 2}}{\sqrt{|E||F|}}} .
$$

From the above inequality, we can easily derive the following analogue of Lemma 2 for the distance set $\Delta_{Q}(E, F)$.

Lemma 5 For any $0<\epsilon<1$. If $|E||F| \geqslant 9 q^{(d-1)+\epsilon}$ then

$$
\Delta_{Q}(E, F) \geqslant \frac{\sqrt{|E||F|}}{3 q^{(d-1) / 2}} \geqslant q^{\epsilon / 2}
$$

for any $q \gg 1$.

By Theorem 7, each graph $E_{q}(d, Q, a)$ is a $\left(q^{d}, q^{d-1} \pm q^{\lfloor(d-1) / 2\rfloor}, 2 q^{(d-1) / 2}\right)$-regular graph. By (9), for any $a \neq 0 \in \mathbb{F}_{q}$, we have

$$
\alpha_{2}\left(E_{q}(d, Q, a)\right) \leqslant\left(\frac{2 q^{(3 d-1) / 2}}{q^{d-1}-q^{(d-1) / 2}}\right)^{2} \leqslant 9 q^{d+1} .
$$

Thus, if $|E||F| \geqslant 9 q^{d+1}$ then $E, F$ is not an independent pair of $E_{q}(d, Q, a)$ for any nonzero $a$. This implies that there exist $x \in E$ and $y \in F$ such that $Q(x, y)=a$ for any $a \in \mathbb{F}_{q}$. We have the following analogue of Lemma 1. 
Lemma 6 Let $E, F \subset \mathbb{F}_{q}^{d}$ such that $|E||F| \geqslant 9 q^{d+1}$. Then $\Delta_{Q}(E, F)=\mathbb{F}_{q}$.

Theorem 5 is immediate from Lemma 5 and Lemma 6 . The proof of Theorem 6 is similar and is left for the readers. Note that the analogue of Lemma 3 for the distance set $\Delta_{H}(E, F)$ is interesting in its own right.

Lemma 7 Let $E, F \subset H_{q}$ such that $|E||F| \geqslant 9 q^{3}$. Then $\left|\Delta_{H}(E, F)\right| \geq q-1$.

\section{$5 \quad$ Further remarks}

The proofs in [17] and [18] show that the conclusion of Theorem 3 holds with the nondegenerate quadratic form $Q$ is replaced by any function $F$ with the property that the Fourier transform satisfies the decay estimates

$$
\left|\hat{F}_{t}(m)\right|=\left|q^{-d} \sum_{x \in \mathbb{F}_{q}^{d: F(x)=t}} \chi(-x . m)\right| \leqslant C q^{-(d+1) / 2}
$$

and

$$
\left|\hat{F}_{t}(0, \ldots, 0)\right|=\left|q^{-d} \sum_{x \in \mathbb{F}_{q}^{d}: F(x)=t} \chi(-x .(0, \ldots, 0))\right| \leqslant C q^{-1},
$$

where $\chi(s)=e^{2 \pi i \operatorname{Tr}(s) / q}$ and $m \neq(0, \ldots, 0) \in \mathbb{F}_{q}^{d}$ (recall that for $y \in \mathbb{F}_{q}$, where $q=p^{r}$ with $p$ prime, the trace of $y$ is defined as $\left.\operatorname{Tr}(y)=y+y^{p}+\ldots+y^{p^{r-1}} \in \mathbb{F}_{q}\right)$. The basic object in these proofs is the incidence function

$$
\begin{aligned}
I_{B, C}(j)=|B||C| v(j) & =|(x, y) \in B \times C: F(x-y)=j| \\
& =\sum_{x, y \in \mathbb{F}_{q}^{d}} B(x) C(y) F_{j}(x-y),
\end{aligned}
$$

where $B, C, F_{j}$ denotes the characteristic function of the sets $B, C$ and $\{x: F(x)=j\}$, respectively. Using the Fourier inversion, we have

$$
I_{B, C}(j)=q^{2 d} \sum_{m \in \mathbb{F}_{q}^{d}} \overline{\hat{B}(m)} \hat{C}(m) \hat{F}_{j}(m) .
$$

Now we define the $F$-distance graph $G_{F}(q, d, j)$ with the vertex set $V=\mathbb{F}_{q}^{d}$ and the edge set

$$
E=\{(x, y) \in V \times V \mid x \neq y, F(x-y)=j\} .
$$

Then the exponentials (or characters of the additive group $\mathbb{F}_{q}^{d}$ )

$$
e_{m}(x)=\exp \left(\frac{2 \pi i \operatorname{Tr}(x \cdot m)}{p}\right)
$$


for $x, m \in \mathbb{F}_{q}^{d}$, are eigenfunctions of the adjacency operator for the $F$-distance graph $G_{F}(q, d, j)$ corresponding to the eigenvalue

$$
\lambda_{m}=\sum_{F(x)=j} e_{m}(x)=q^{d} \hat{F}_{j}(-m)
$$

Thus, the decay estimates (22) and (23) are equivalent to

$$
\lambda_{m} \leqslant C q^{(d-1) / 2}
$$

for $m \neq(0, \ldots, 0) \in \mathbb{F}_{q}^{d}$, and

$$
\lambda_{(0, \ldots, 0)} \leqslant C q^{d-1} .
$$

Let $A$ be the adjacency matrix of $G_{F}(q, d, j)$ with the orthonomal base $v_{0}, \ldots, v_{q^{d}-1}$, corresponding to eigenvalues $\lambda_{(0, \ldots, 0)}, \ldots, \lambda_{(q-1, \ldots, q-1)}$, where $v_{0}=\overline{1} / \sqrt{n}$. For any two sets $B, C \subset \mathbb{F}_{q}^{d}$, let $v_{B}$ and $v_{C}$ be the characteristic vectors of $B$ and $C$. Let $v_{B}=\sum_{i} \beta_{i} v_{i}$ and $v_{C}=\sum_{i} \gamma_{i} v_{i}$ be their representations as linear combinations of $v_{0}, \ldots, v_{q^{d}-1}$. We have

$$
\begin{aligned}
I_{B, C}(j)=e_{G_{F}(q, d, j)}(B, C) & =v_{B} A v_{C} \\
& =\left(\sum_{i} \beta_{i} v_{i}\right) A\left(\sum_{j} \gamma_{j} v_{j}\right) \\
& =\left(\sum_{i} \beta_{i} v_{i}\right)\left(\sum_{j} \gamma_{j} \lambda_{j} v_{j}\right) \\
& =\sum_{i} \lambda_{i} \beta_{i} \gamma_{i} .
\end{aligned}
$$

From (24), (26) and the above expression, we can see the similarity between our approach and those in [17] and [18] as follows. Given the decay estimates (22) and (23), we can bound the incidence function as in [17] and [18]

$$
\begin{aligned}
I_{B, C}(j) & \leqslant|B||C| \hat{F}_{j}(0, \ldots, 0)+q^{(d-1) / 2} \sum_{m \neq(0, \ldots, 0)} q^{d}|\hat{B}(m)||\hat{C}(m)| \\
& \leqslant C q^{-1}|B||C|+C q^{(d-1) / 2} q^{d}\left(\sum_{m \neq(0, \ldots, 0)}|\hat{B}(m)|^{2}\right)^{1 / 2}\left(\sum_{m \neq(0, \ldots, 0)}|\hat{C}(m)|^{2}\right)^{1 / 2} \\
& \leqslant C q^{-1}|B||C|+C q^{d-1}\left(\sum_{x}|B(x)|^{2}\right)^{1 / 2}\left(\sum_{x}|C(x)|^{2}\right)^{1 / 2} \\
& \leqslant C q^{-1}|B||C|+C q^{d-1} \sqrt{|B|} \sqrt{|C|} .
\end{aligned}
$$

Given the bounds (27), (28) for eigenvalues of the $F$-distance graph $G_{F}(q, d, j)$, we 
obtain the same bound for the incidence function

$$
\begin{aligned}
I_{B, C}(j) & =\lambda_{(0, \ldots, 0)}\left\langle v_{B}, \overline{1} / \sqrt{q^{d}}\right\rangle\left\langle v_{C}, \overline{1} / \sqrt{q^{d}}\right\rangle+\sum_{m \neq(0, \ldots, 0)} \lambda_{m} \beta_{m} \gamma_{m} \\
& \leqslant C q^{-1}|B \| C|+C q^{(d-1) / 2} \sum_{m \neq(0, \ldots, 0)}\left|\beta_{m}\right|\left|\gamma_{m}\right| \\
& \leqslant C q^{-1}\left|B\left\|C \mid+C q^{(d-1) / 2}\right\| \beta\left\|_{2}\right\| \gamma \|_{2}\right. \\
& =C q^{-1}|B \| C|+C q^{(d-1) / 2} \sqrt{|B|} \sqrt{|C|} .
\end{aligned}
$$

Thus, our approach and the Fourier methods in [18] and [17] are almost identical. Many results obtained from the Fourier method can be proved using our method and vice versa. However, both methods have their own advantages. On one hand, many results (obtained from the Fourier methods) in [16] are hard to derive from the graph theory method. On another hand, the graph theory method sometimes gives us many simple applications without invoking more advanced tools like the character sums or Fourier transform (see [30]).

It is worth to notice that Theorem 4 and Theorem 6 also follow from the Fourier methods. However, we will need to use Soto-Andrade sums bound instead of Kloosterman sums bounds for non-Euclidean spaces. We will address these results in a consequent paper.

Finally, the $F$-distance graph with the function $F$ satisfying the decay estimates (22) and (23) give us a possible approach to construct triangle-free graphs with very high chromatic number. For example, if we can find a sum-free variety in $\mathbb{F}_{q}^{d}$ defined by a polynomial $F\left(x_{1}, \ldots, x_{d}\right) \in \mathbb{F}_{q}\left[X_{1}, \ldots, X_{d}\right]$ (i.e., $F(X)=0, F(Y)=0$ then $F(X+Y) \neq 0$ for every $\left.X, Y \in \mathbb{F}_{q}^{d}\right)$ then we can construct a triangle-free graph of order $n=q^{d}$ with the chromatic number at least $C n^{(d-1) / 2 d}$. We see in Section 3 that the varieties of degree two

only give us triangle-free graphs over vector spaces of dimension two. We hope to address this problem for higher dimensional vector spaces in a subsequent paper.

\section{Acknowledgments}

The author is very grateful to Dang Phuong Dung and Si Li for many useful discussions, helpful comments and endless encouragement. He also would like to thank Professor Alex Iosevich for constructive comments on early version of this manuscript and the referee for helpful suggestions to improve the presentation of this paper.

\section{References}

[1] N. Alon, Explicit Ramsey graphs and orthonormal labellings, The Electronic Journal of Combinatorics 1 (1994), R12, 8pp.

[2] N. Alon, Tough Ramsey graphs without short cycles, Journal of Algebraic Combinatorics

[3] N. Alon, J.H. Spencer, The Probabilisitic Method, 2nd ed., Wiley-Interscience, 2000. 
[4] E. Bannai, W.M. Kwok, S.-Y. Song, Ennola type dualities in the character tables of some association schemes, Mem. Fac. Sci. Kyushi Univ. Ser. A. 44 (1990), 129-143.

[5] E. Bannai, S. Hao, S.-Y. Song, Character tables of the association schemes of finite orthogonal groups acting on the nonisotropic points, Journal of Combinatorial Theory Series A 54 (1990), 164-170.

[6] E. Bannai, S. Hao, S.-Y. Song, H. Wei, Character tables of certain association schemes coming from finite unitary and sympletic groups, Journal of Algebra 144 (1991), 189200.

[7] E. Bannai, O. Shimabukuro, H. Tanaka, Finite analogues of non-Euclidean spaces and Ramanujan graphs, European Journal of Combinatorics 25 (2004), 243-259.

[8] E. Bannai, O. Shimabukuro, H. Tanaka, Finite Euclidean graphs and Ramanujan graphs, Discrete Mathematics (to appear).

[9] D. Bauer, J. Vandenheuvel and E. Schmeichel, Toughness and Triangle-Free Graphs, Journal of Combinatorial Theory, Series B 65 (2) (1995), 208-221.

[10] J. Bourgain, Hausdorff dimension and distance sets, Israel Journal of Mathematics 87 (1994), 1993-201.

[11] J. Bourgain, N. Katz, T. Tao, A sum-product estimate in finite fields, and applications, Geom. Funct. Anal. 14 (2004), 27-57.

[12] V. Chvátal, Tough graphs and hamiltonian circuits, Discrete Mathematics 5 (1973), 215-218.

[13] J. Gimbel and C. Thomassen, Coloring triangle-free graphs with fixed size, Discrete Mathematics 219 (2000), no. 1-3, 275-277.

[14] D. Hart, A. Iosevich, J. Solymosi, Sum-product estimates in finite fields via Kloosterman sums, International Mathematics Research Notices (to appear).

[15] D. Hart, A. Iosevich, Sums and products in finite fields: an integral geometric viewpoint, preprint, 2007.

[16] D. Hart, A. Iosevich, D. Koh and M. Rudnev, Averages over hyperplanes, sumproduct theory in vector spaces over finite fields and the Erdös-Falconer distance conjecture, preprint, 2007.

[17] A. Iosevich, D. Koh, Erdős-Falconer distance problem, exponential sums, and Fourier analytic approach to incidence theorems in vector spaces over finite fields, preprint, 2007.

[18] A. Iosevich, M. Rudnev, Erdős distance problem in vector spaces over finite fields, Transactions of the American Mathematical Society 359 (12) (2007), 6127-6142.

[19] N.M. Katz, Estimates for Soto-Andrade sums, J. Reine Angew. Math. 438 (1993), 143-161.

[20] N.M. Katz, A note on exponential sums, Finite Fields Appl. 1 (1995), 395-398.

[21] J. H. Kim, The Ramsey number $R(3, t)$ has order to magnitude $t^{2} / \log t$, Random Structure Algorithms 7 (1995), 173-207. 
[22] W.M. Kwok, Chracter table of association schemes of affine type, European Journal of Combinatorics, 13 (1992), 167-185.

[23] W.-C.W. Li, Number Theory with Applications, World Scientific, River Edge, NJ, 1996.

[24] A. Lubotzky, R. Phillips and P. Sarnak, Ramanujan graphs, Combinatoria 8 (1988), 261-277.

[25] A. Medrano, P. Myers, H.M. Stark and A. Terras, Finite analogues of Euclidean space, Journal of Computational and Applied Mathematics, 68 (1996), 221-238.

[26] I. E. Shparlinski, On the set of distances between two sets over finite fields, International Journal of Mathematics and Mathematical Sciences 2006, Article ID 59482, 1-5.

[27] T. Tao, Finite field analogues of Erdős, Falconer, and Furstenberg problems, preprint.

[28] A. Terras, Survey of Spectra of Laplacians on Finite Symmetric Spaces, Experimental Mathematics, (1996).

[29] L. A. Vinh, Some coloring problems for unit-quadrance graphs, The proceedings of Australian Workshop on Combinatorial Algorithms, 2006, 361-367.

[30] L. A. Vinh, Szemerédi-Trotter theorem and sum-product estimate in finite fields, preprint, 2007.

[31] L. A. Vinh and D. P. Dung, Explicit tough Ramsey graphs, preprint, 2007.

[32] V. Vu, Sum-Product estimates via directed expanders, preprint, 2007. 